\title{
Research article \\ The effect of deep breathing test on heart rate variability in obese and non-obese patients with myocardial infarction
}

\author{
Srinath C. Galag ${ }^{1}$, Rajalakshmi R. ${ }^{2}$, Nagaraj Desai ${ }^{3}$, Basavanagowdappa $\mathrm{H}^{4}$ \\ ${ }^{1}$ Assistant Professor, ${ }^{2}$ Professor and Head, Department of Physiology, JSS Academy of Higher Education and Research, Sri \\ Shivarathreeshwara Nagar, Mysuru-570015, Karnataka, India \\ ${ }^{3}$ Adjunct Professor of Cardiology, ${ }^{4}$ Professor of General Medicine, JSS Academy of Higher Education and Research, JSS \\ Hospital, MG Road, Mysuru-570004, Karnataka, India
}

(Received: December $2020 \quad$ Revised: February $2021 \quad$ Accepted: March 2021)

Corresponding author: Srinath C. Galag. Email: srinathcg @jssuni.edu.in

\begin{abstract}
Introduction and Aim: Obesity and metabolic syndrome increases the risk of cardiovascular diseases (CVD). The acute myocardial infarction may be associated with autonomic dysfunction and it may have a bearing on the prognosis. The objective of the study was to examine the effect of deep breathing test on heart rate variability in obese and non-obese patients with myocardial infarction (MI).

Materials and Methods: The patients with acute myocardial infarction were confirmed by universal definition and treatment was administered. At 12 weeks of follow up, the MI patients were screened and divided into two groups. 30 patients of MI with BMI 25 to $30 \mathrm{~kg} / \mathrm{m}^{2}$ formed the obese group. 30 patients of MI with BMI 18.5 to $24.9 \mathrm{~kg} / \mathrm{m}^{2}$ formed the non-obese group. The baseline ECG was taken for 5 minutes by using Niviqure B3 machine for HRV analysis. Then, deep breathing test (DBT) was performed in a standardized manner.

Results: The two groups were well matched for the age (53.7 \pm 11.5 vs. $55.2 \pm 9.2$ years). In the baseline there was a statistically significant decrease in the total power (TP) of the heart i.e., HF plus LF $\left(2178 \pm 762 \mathrm{~ms}^{2}\right.$ vs $2991 \pm 771 \mathrm{~ms}^{2}$ with a ' $p$ ' value 0.001 ) in the obese group when compared to non-obese group. On DBT there was a significant decrease in heart rate $(81 \mathrm{bpm}$ to $65 \mathrm{bpm})$ in non-obese group. However, in obese group there was no significant decrease in heart rate (79bpm to $72 \mathrm{bpm})$ on DBT.
\end{abstract}

Conclusion: Accordingly, we conclude that in obese patients with MI there is a reduction in respiratory vagal modulation of heart rate during DBT.

Keywords: Obesity; heart rate variability, myocardial infarction.

\section{INTRODUCTION}

$\mathrm{O}$ besity and metabolic syndrome increase the risk of cardiovascular diseases (CVD) such as ischemic heart disease (IHD). Myocardial infarction (MI) is one of the commonest clinical manifestations IHD. The underlying mechanisms may be many and cardiac autonomic nervous system (ANS) dysfunction is also considered as one among them. The ANS activity and imbalance between its two main components (parasympathetic and sympathetic nervous control) are important factors contributing to the initiation and progression of many CVD's such as coronary artery disease, ventricular arrhythmia, arterial hypertension, left ventricular hypertrophy, and cardiomyopathy related to obesity (1).

To assess cardiovascular autonomic nervous system changes in obesity, heart rate variability (HRV) analysis is performed. Respiratory sinus arrhythmia occurs in humans from the central nervous system mechanism i.e., the connection of respiratory and cardiac control centres (1).The heart rate increases during inspiration due to decrease vagal activity and decreases during expiration due to increased vagal activity. HRV is defined as the fluctuation in degree of heart rate around its mean value. HRV is mirroring the regularity of heart beats i.e., bigger regularity means lowers HRV (and vice versa). In the HRV, high frequency (HF) spectral power corresponds to the magnitude of respiratory related heart rate oscillations i.e. respiratory sinus arrhythmia (RSA). HF frequency is often analysed as it is an index of phasic parasympathetic activity (1). Several studies have demonstrated that there is lower parasympathetic activity (lower HF HRV power) in obesity (1-5).

In patients with an acute MI the absence of respiratory sinus arrhythmias is associated with an increase in 'in-hospital' mortality (6). Depressed HRV is a powerful predictor of mortality and of arrhythmic complications (e.g. symptomatic sustained ventricular tachycardia) in patients following acute MI (6). The autonomic tone and reflexes after myocardial infarction (ATRAMI) trial was a multicentre observational study performed. ATRAMI provides clinical evidence that after myocardial infarction the analysis of HRV and vagal reflexes has significant prognostic value (7). Another similar study confirmed that heart rate variability 
and heart rate turbulence yield more powerful prognostic information for arrhythmic events when measured late (6-10weeks after AMI) rather than early (within 2weeks) after AMI (8). Thus the lack of recovery from reduced autonomic function after AMI is a stronger predictor of adverse events than decreased autonomic function at the time of AMI (8). Therefore, our objective was to compare the HRV in obese and non-obese patients with AMI. In order to explain the heart rate changes with respiration (RSA) in obesity, we undertook this work with a different approach i.e., effect of deep breathing test (respiratory vagal modulation) on HRV. The aim of the study was to examine the effect of deep breathing test on heart rate variability (HRV) in obese and non-obese patients with myocardial infarction.

\section{MATERIALS AND METHODS}

The study period was from November 2015 to October 2019. The study design was case control comparative study. The institutional ethical committee of JSS Medical College, Mysuru (JSSMC/IEC/10/5553/2016-17 dated 09/12/2016), approved this study. The patients with acute myocardial infarction were confirmed by universal definition (symptoms, cardiac enzymes and electrocardiographic changes) and treatment was administered in JSS Hospital Mysuru. During followup at 12 weeks, in the Cardiology department, about 200 patients with MI were screened. The present study included 60 patients of post myocardial infarction patients, having the following criteria, were selected for recording the data.

\section{Inclusion criteria}

Patients were considered eligible for the study if their age was more than 35 and less than 70 years. The patients with acute myocardial infarction (first or consequent) who survived to hospital discharge till 12 weeks were included for the study. The Myocardial infarction as confirmed by third universal definition (symptoms, cardiac enzymes and electrocardiographic changes-as per WHO definition) (9).

\section{Exclusion criteria}

Patients were considered not eligible, if the patient's age was less than 35 years or more than 70 years. The patients participating in any other study projects in the hospital were also not included. Similarly, any other critical illness or cardiac ailment which limits the life of the patient less than one year such as, severe valvular diseases, recurrent ventricular arrhythmias, heart failure, such patients were excluded.

The patients who met the above inclusion criteria were grouped based on BMI according to the Asianpacific cutoff points normal weight (18.5-22.9 $\mathrm{kg} / \mathrm{m} 2$ ), overweight (23-24.9 kg/m2), and obese (25$30 \mathrm{~kg} / \mathrm{m}^{2} ; 10$ ). Informed consent was obtained from all the subjects. Diabetes education and exercise prescription was given to the diabetic patients. Body mass index was calculated by recording height, weight and applying the formula,

Body mass index $=\frac{\text { Weight in kilograms }}{\text { Height in meters square }}$

(1) 30 obese patients aged 35-70 years with myocardial infarction (at 12 weeks) having BMI 25 to $30 \mathrm{~kg} / \mathrm{m}^{2}$ formed the obese group.

(2) 30 non-obese patients aged 35-70 years with myocardial infarction (at 12weeks) having BMI 18.5 to $24.9 \mathrm{~kg} / \mathrm{m}^{2}$ formed the non-obese \{normal weight $(18.5-22.9 \mathrm{~kg} / \mathrm{m} 2)$ and overweight $(23-24.9 \mathrm{~kg} / \mathrm{m} 2)$ \} group.

This sample size was estimated to be enough to detect a clinically relevant difference of $10 \%$ in the HRV parameters at $5 \%$ level of significance with $80 \%$ power. The Physiological parameters included were Weight, Height, BMI, Heart rate, Blood pressure, Resting ECG for HRV, Deep breathing test.

\section{Materials used in the study}

Niviqure B3 HRV analysis machine with software, cardiac monitors, automated sphygmomanometer, weighing machine available in the cardiac autonomic function test lab (AFT) in the Cardiology department, JSSH, Mysuru were used. The Biochemical tests, such as FBS, PPBS, HbA1c, Lipid profile, CK-MB and Troponin $\mathrm{T}$, were done using standard protocols used in biochemistry labs.

\section{Procedure of $\mathrm{HRV}$ recording and analysis}

\section{1) Baseline recording of $\mathrm{HR}, \mathrm{BP}$}

The cardiac autonomic function tests were carried out in the AFT lab in the department of Cardiology between 10AM and 1.00 PM under ambient conditions after having made the patients comfortable and relaxed. The patient was asked to take rest for 15 minutes in supine position. The baseline HR and BP were recorded by using Niviqure B3 HRV machine and automated sphygmomanometer. A total of 200 patients ECG recording was done. The normal ECG's having sinus rhythm were included for the study. The baseline heart rate was analyzed for frequency (HF, LF, TP) domains parameters (6) using the Niviqure software and tabulate as shown in below tables. The ECG's having abnormalities such as atrial fibrillation, heart blocks etc. were excluded because such ECG's analysis gives false high/low values of HRV (8).

\section{2) Deep breathing test}

The patient was asked to rest in supine position for 5 minutes. Then he/she was asked to breathe deeply at a rate of 6 breaths per minute (allowing 5 seconds for inspiration and 5 seconds for expiration). The heart rate was calculated using the Niviquere ECG 
machine. The maximum and minimum heart rate with each breath was calculated (1, 11-13). The E/I ratio was also calculated. E/I ratio is the ratio of longest RR interval during expiration to the shortest RR interval during inspiration.

Table 1: Frequency domain indices of HRV

\begin{tabular}{|c|c|c|}
\hline $\begin{array}{c}\text { Total Power }\left(\mathbf{m s}^{\mathbf{2}}\right): \\
\text { Frequency range } \\
\mathbf{0 . 4 H z}\end{array}$ & $\begin{array}{c}\text { The variance of RR intervals over the } \\
\text { temporal segment }\end{array}$ & $\begin{array}{c}\text { Overall vagal potency of cardiac } \\
\text { modulation }\end{array}$ \\
\hline $\mathrm{VLF}\left(\mathrm{ms}^{2}\right): 0-0.04 \mathrm{~Hz}$ & Very low frequency power of HRV & Integrity of renin angiotensin system \\
\hline $\mathrm{LF}\left(\mathrm{ms}^{2}\right): 0.04-0.15 \mathrm{~Hz}$ & Low frequency power of HRV & Mainly, cardiac sympathetic drive \\
\hline $\mathrm{HF}\left(\mathrm{ms}^{2}\right): 0.15-0.4 \mathrm{~Hz}$ & High frequency power of HRV & Cardiac parasympathetic drive \\
\hline $\mathrm{LF} / \mathrm{HF}$ ratio & Ratio of LF to HF power & Sympathovagal balance \\
\hline
\end{tabular}

\section{Statistical methods}

The data obtained was tested for normality. By applying Kolmogorov-smirnov and Shapiro-wilk test to the data it is checked for the normality distribution for each parameter. The data is normally distributed. The following statistical tests done accordingly.

1. Mean and S.D. (standard deviation) of all the cardiovascular parameters, in both the groups (stated above) were calculated.
2. Student's t - test (un-paired) was applied at $5 \%$ level of significance to test the significance of changes in cardiovascular parameters stated above.

3. Correlation analysis between Body mass index and Total power of HRV was done.

\section{RESULTS}

Table 2: The characteristics of the patients with myocardial infarction

\begin{tabular}{|c|c|c|c|}
\hline Groups & Non-obese & Obese & 'p' value \\
\hline Total no. of patients & 30 & 30 & - \\
\hline Age (in years) & $53 \pm 11$ & $55 \pm 9$ & 0.4 \\
\hline No. of female patients & 08 & 05 & - \\
\hline Smokers & 13 & 12 & - \\
\hline BMI kg/m & $23.5 \pm 1.1$ & $27.8 \pm 1.1$ & $0.005^{*}$ \\
\hline HbA1C (gm/dl) & $7.3 \pm 1.5$ & $7.48 \pm 2.1$ & 0.4 \\
\hline CK MB (ng/ml) & $21.7 \pm 06$ & $23.0 \pm 20.5$ & 0.3 \\
\hline Troponin T (ng/ml) & $1.6 \pm 0.6$ & $0.3 \pm 0.2$ & 0.8 \\
\hline $\begin{array}{c}\text { Total Cholesterol } \\
(\mathrm{mg} / \mathrm{dl})\end{array}$ & $175 \pm 29$ & $185 \pm 40$ & 0.6 \\
\hline LDL (mg/dl) & $118 \pm 28$ & $119 \pm 33$ & 0.7 \\
\hline HDL (mg/dl) & $58 \pm 14$ & $66 \pm 19$ & 0.6 \\
\hline
\end{tabular}

Table 3: The HRV test in obese and non-obese patients with MI

\begin{tabular}{|c|c|c|c|}
\hline BMI & Non-Obese & Obese & 'p'Value \\
\hline No. of patients & 30 & 30 & - \\
\hline $\begin{array}{c}\text { RR interval } \\
\text { (mean } \pm \text { S.D.) }\end{array}$ & $848 \pm 133$ & $849 \pm 100$ & 0.93 \\
\hline LF & $942 \pm 195$ & $756 \pm 205$ & $0.01^{*}$ \\
\hline HF & $1926 \pm 667$ & $1270 \pm 425$ & $0.01^{*}$ \\
\hline Total Power $\left(\mathrm{ms}^{2}\right)$ & $2991 \pm 771$ & $2178 \pm 762$ & $0.001^{*}$ \\
\hline LF/HF & 0.50 & 0.60 & - \\
\hline
\end{tabular}

Table 4: The effect DBT on HRV in obese and non-obese patients with MI

\begin{tabular}{|c|c|c|c|}
\hline BMI & Non-Obese & Obese & 'p' value \\
\hline No. of patients & 30 & 30 & - \\
\hline LF/HF on DBT & 0.30 & 0.50 & - \\
\hline Mean HR in bpm (MAX) during DBT & 81 & 79 & - \\
\hline Mean HR in bpm (MIN) during DBT & 65 & 70 & - \\
\hline HR (MAX-MIN diff.) & 16 & 8 & - \\
\hline RR interval in mean \pm S.D. (MAX) during DBT & $971 \pm 107$ & $886 \pm 95$ & $0.002^{*}$ \\
\hline RR interval in mean \pm S.D. (MIN) during DBT & $694 \pm 87$ & $720 \pm 99$ & 0.29 \\
\hline E/I during DBT & 1.41 & 1.23 & $0.001 *$ \\
\hline
\end{tabular}


$*<0.05$

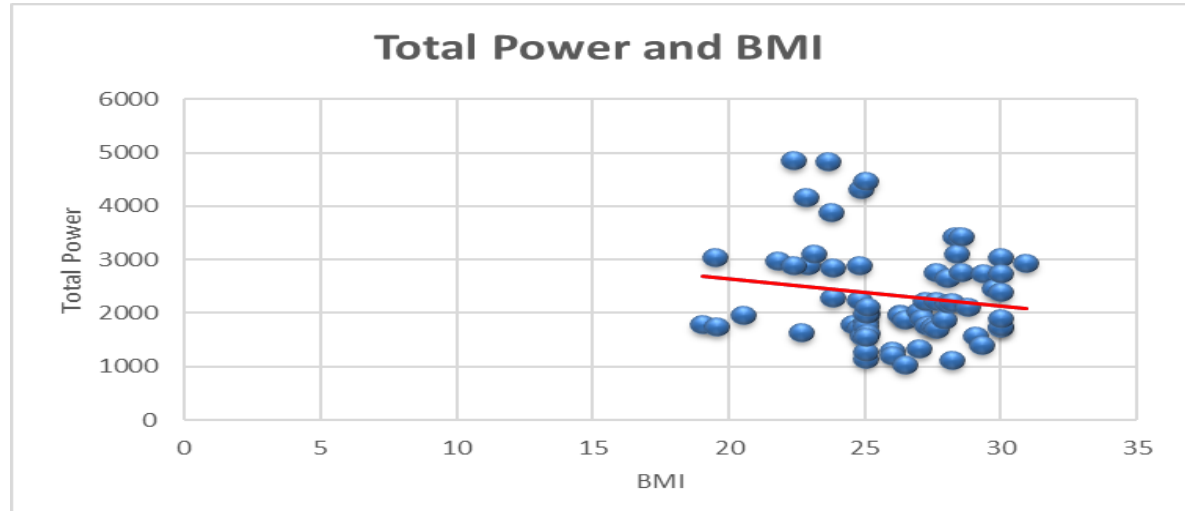

Graph 1: Correlation between Total power (frequency domain in $\mathrm{ms}^{2}$ ) and BMI

There was no significant difference in mean and standard deviation of age between the two groups $(53.7 \pm 11.5$ vs. $55.2 \pm 9.2$ years). The baseline total power of the heart $\left(2178 \pm 762 \mathrm{~ms}^{2}\right.$ vs $2991 \pm 771 \mathrm{~ms}^{2}$ with a 'p' value 0.001 ) was a significantly less in the obese group, when compared to non-obese group as shown in table 3 . There was a negative correlation ($0.16)$ between BMI and Total power of HRV as shown in the above graph 1.On DBT there was a significant decrease in heart rate (from $81 \mathrm{bpm}$ to $65 \mathrm{bpm})$ in non-obese group. However, in obese group there was no significant decrease in heart rate (from $79 \mathrm{bpm}$ to $70 \mathrm{bpm}$ ) on DBT. E/I ratio was 1.41 in non-obese group and 1.23 in obese group as shown in table 4.

\section{DISCUSSION}

The main findings of our study are the total power of heart rate variability (HRV) is reduced in obese group of patients recovering from myocardial infarction when compared to non-obese group of patients. Here, both the group of patients are matched for age, sex and number of smokers as shown in table 2. In both the groups of patients type 2 diabetes mellitus, dyslipidemia and hypertension were under control. Previous studies have shown that in patients with myocardial infarction there is decrease in $\operatorname{HRV}(8,14-16)$.

Abnormality of HRV reflects independent prognostic information in patients with postmyocardial infarction. Depressed HRV after an MI may reflect a decrease in vagal activity (HF component) directed to the heart, which leads to prevalence of increase in sympathetic activity (LF component of HRV as shown in table 1) and to cardiac electrical instability (6). Extremely low values are associated with a lack of autonomic modulation of heartrate (8). In our study, we examined the respiratory sinus arrhythmia (RSA) mechanisms by comparing the effect of DBT on HRV in obese and non-obese patients with MI. The heart rate increases during inspiration due to decrease vagal activity and decreases during expiration due to increased vagal activity i.e., HRV changes with respiration. During deep breathing, there is more expansion of lungs and thoracic cage, causing increased nerve traffic from the proprioceptive receptors of thoracic cage. This causes activation of hypothalamic and medullary cardio vagal centre $(1,11,17)$. This increased cardiac vagal activity (HF component of $\mathrm{HRV}$ as shown in table 1) causes decrease in heart rate and restores autonomic balance. The results of our study show that on DBT there was a significant decrease in heart rate in non-obese group. However, in obese group there was no significant decrease in heart rate on DBT. E/I ratio was 1.41 in non-obese group and 1.23 in obese group as shown in table 4. All these clinical studies together, explain that there are reductions in cardiac parasympathetic regulation in the obese patients with MI.

The alterations in the morphology of the heart due to obesity could be the precursor to the decreased HRV $(1,2)$. A recent study done in 2019 by Ilan Goldenberg et.al. The HRV-DETECT (Heart Rate Variability for the Detection of Myocardial Ischemia) is the first study done to evaluate HRV testing for the detection of myocardial ischemia. It showed that short-term HRV analysis using digital technology can be used for improved risk assessment for myocardial ischemia in individuals without known CAD (18). The modifiable risk factors for ischaemic heart disease are hypertension, type 2 diabetes mellitus, smoking and obesity. Several previous studies have found that obesity causes development of hypertension and type 2 diabetes mellitus (1-5). A comparative study done in 2017 by Jayesh DS.et.al in Indian patients, found that type 2 diabetics with or without hypertension had in general reduced total power, reduced time domain and frequency domain parameters of HRV (19). They found that there was no difference in overall cardiac autonomic status in hypertensive diabetics as compared to normotensive diabetics. In their study, they did not take body mass index into the comparison. Therefore, in our study we focused on the obesity. This is because excess weight induces ANS dysfunction, which may be involved in the haemodynamic and metabolic alterations such as hypertension, insulin resistance and dyslipidaemia 
that increase the cardiovascular risk of obese individuals $(20,21)$.

In obesity, the excess adipose tissue secretes adipokines (leptin and adiponectin) hormone like peptides, which have an impact on glucose and lipid metabolism, inflammatory process and other bioactivities. The relation between blood adipokines concentration to the HRV parameters may indicate a possible link between adipokines and disturbances of the autonomic nervous system (21). Further, the studies have shown that weight loss is able reverse the metabolic and autonomic alterations associated with obesity (20).

The macrophages are present in a large proportion as the immune cells in perivascular adipose tissue (PVAT). In lean person's adipose tissue, the macrophage phenotype is predominantly M2 macrophages (alternatively activated macrophages) which release anti-inflammatory cytokines such as interleukin-10.However, in obese person's adipose tissue there is a phenotypic switch from M2 macrophages to M1 macrophages (classically activated macrophages).M1 macrophages are the main source of TNF- $\alpha$ and IL-6. It is worth noting that in obesity adipocyte-derived M1 macrophages may contribute directly to vascular dysfunction by reducing bioavailability of the vasodilators in the endothelium (22).

The adverse impact of obesity on heart rate variability is modified by a NFE2L2 (nuclear factor erythroid 2-like 2; also known as NRF2) gene variant (23). This study showed that there is inverse relationship between BMI and HRV. There is increasing evidence that Nrf2 is a key regulator of cardiovascular homeostasis by suppressing oxidative stress such as reactive oxygen species (ROS). Nrf2 is important to preserve a healthy endothelial phenotype and to maintain the functional integrity of vasculature.

\section{Limitations of the study}

There were few limitations in our study. First, it is a cross-sectional study. A longitudinal study with a larger sample can be done. Second, we used 5minute HRV analysis that reveals only short-term changes in cardiac autonomic status. The other tests for autonomic function such as tilt-table test, valsalva manoeuvre test and 24-hour HRV test were not done.

\section{CONCLUSION}

This study shows that obesity with myocardial infarction, results in a reduction heart rate variability. The results of our study support that there is dysfunction in parasympathetic (HF) component of autonomic nervous system in obese group, as there is decreased respiratory vagal modulation of heart rate during deep breathing test when compared to non-obese group. Thus, HRV test analysis may be useful in diagnosing the cardiac autonomic dysfunction in obese patients with myocardial infarction.

\section{ACKNOWLEDGEMENT}

Authors acknowledge valuable contribution to the scientific content given by Dr. P.V. Salimath, Advisor (Research), JSS Medical College, Mysuru. Source of Data: Yoga Care Clinical Trial in the Department of Cardiology JSS Hospital, Mysuru.

\section{CONFLICT OF INTEREST}

Authors have no conflict of interest.

\section{REFERENCES}

1. Javorka, M., Krohova, J., Czippelova, B., Turianikova, Z., Magzotova, N., Wistz, R. et.al. Respiratory sinus arrhythmia mechanisms in young obese subjects. Frontiers in Neuroscience. 2020; (14) 204:1-8.

2. Liao, D., Rodriguez-Colon, Fan He, Bixler. Childhood Obesity and autonomic dysfunction: Risk for cardiac morbidity and mortality. Curr Treat Options Cardiovasc Med. 2014; 16(10): 342-358.

3. Srinath, C. G., Rajalaksmi, R., Nataraj, S.M. Heart rate QT and QTc intervals response to isometric handgrip exercise test in obese and normal weight subjects. Biomedicine. 2010; 30(3): 306-310.

4. Rajalakshmi, R., VijayaVageesh, Y., Nataraj, S.M, Murali Dhar, Srinath, C. G. Heart rate variability in Indian obese young adults. Pak J Physiol. 2012; 8(1): 39-44.

5. Archana, D., Balasubramanian, K. Autonomic dysfunction in central obesity. World J. Med. Sci. 2013; 8 (2): 118-122.

6. Guidelines.Task force of the European society of Cardiology and North American society of pacing and Electrophysiology. Heart rate variability - standards of measurement, physiological interpretation and clinical use. Circulation. 1996; 93(5): 1043-1065.

7. La Rovere, M. T., Bigger, J. T., Marcus, F. I., Moratara, A., Schwartz, P. J. ATRAMI (Autonomic Tone and Reflexes After Myocardial Infarction) Investigators. Baroreflex sensitivity and heart-rate variability in prediction of total cardiac mortality after myocardial infarction. Lancet. 1998; 351 (9101); 478-484.

8. Heikki, V. H. Phyllis, K. S. Clinical application of heart rate variability after acute myocardial infarction. Frontiers in Physiology. 2012 ;(3)41: 1-5.

9. Kristian, T., Joseph, S. A., Allan, S. J, Maarten, L. S., Bernard, R. C., Harvey, D. W. Third universal definition of myocardial infarction. Circulation. 2012; 126: 2020-2035.

10. Jeong, U. L., Jae, H. L., Ju, S. K., Young, L. H., Tae, H. K., Seong, H. L., et al., Comparison of World Health Organization and Asia-Pacific body mass index classifications in COPD patients. International Journal of COPD. 2017; 12: 2465-2475.

11. B. Grrishma, Gaur, G. S., Latha, C. S., Velkumary, Senthil Kumar, S., and Gurunandan, U. Assessment of cardiovascular autonomic functions and baroreceptor reactivity in women with premenstrual syndrome. Indian J Physiol Pharmacol. 2015; 59(2): 148-154.

12. Pal, G. K, Pravati, P. Autonomic function tests and spectral analysis of heart rate variability. In: Textbook of Practical Physiology; 4th ed. Hyderabad: Universities Press. 2016: 304-319.

13. Pal, G. K. Functional organization autonomic nervous system. In: Textbook of Medical Physiology; $1^{\text {sted. New }}$ Delhi; Jaypee Brothers Medical Publishers. 2017: 288-324.

14. Juliia, B., Victor, L., Andrii, K., Iryna, L. Normalization of heart rate variability with taurine and meldonium complex 
in post-infarction patients with type 2 diabetes mellitus. Journal of Medicine and Lif 2019; 12(3): 290-295.

15. Antonio, I. T., Anna, V., Michela, A.P.C., Fiorenzo, M., Stefono, V., Marcilleno, M., et al., Heart rate variability is reduced in underweight and overweight healthy adult women. Clinical Physiology Functional Imaging. 2017; 37(2):162-167.

16. Huang, W., Boyle, N., Vaseghi, M. Cardiac innervation and the autonomic nervous system in sudden cardiac death. Card Electrophysiol Clin. 2017; 9(4): 665-679.

17. Ekta, K., Ashok Kumar, J., Deepak, K. Pattern and prevalence of cardiovascular autonomic neuropathy in diabetics visiting a tertiary care referral center in India. Indian J Physiol Pharmacol. 2011; 55 (2): 119-127.

18. Ilan, G., Ronen, G., Nir, S., Michal, E., Jacob, L., Raphael, K., et al., Heart Rate Variability for Risk Assessment of Myocardial Ischemia in Patients without Known Coronary Artery Disease: The HRV-DETECT (Heart Rate Variability for the Detection of Myocardial Ischemia) Study. J Am Heart Assoc. 2019; 8: 1-16.

19. Jayesh, D. S., Sanket, D. B., Hemant, B. M., Sunil, J. P., Bhakti, P. G. Comparative study of cardiac autonomic status by heart rate variability between under-treatment normotensive and hypertensive known type 2 diabetics. Indian Heart Journal. 2017; 69: 52-56.

20. Daniela, G., Monica, N., Giorgio, I., Stefano, T., Rosa. M. B. The Role of the Autonomic Nervous System in the Pathophysiology of Obesity. Review. Frontiers in Physiology. 2017; 8: 1-16.

21. Katarzyna, P., Katarzyna, L., Małgorzata, L., Jerzy, K. W., Jan, H. G. Obesity and heart rate variability in men with myocardial infarction. Cardiology Journal 2008; 15 (1): 4349.

22. Sophie, N. S, Sarah, B. W., Anthony, M. H. Emerging roles of sympathetic nerves and inflammation in perivascular adipose tissue. Cardiovascular Drugs and Therapy. 2019; 33: 245-259.

23. Martin, A., Medea, I., Emmanuel, S., Eva, B., Florian, K., Maco, P. et al., The adverse impact of obesity on heart rate variability is modified by a NFE2L2 gene variant: The SAPALDIA cohort. International Journal of Cardiology. 2017; 228: 341-346. 